\title{
A randomized controlled trial of disclosing genetic risk information for Alzheimer disease via telephone
}

\author{
Kurt D. Christensen, $\mathrm{PhD}^{1,2}$, Wendy R. Uhlmann, MS, $\mathrm{CGC}^{3}$, J. Scott Roberts, PhD ${ }^{4}$, \\ Erin Linnenbringer, $\mathrm{MS}, \mathrm{PhD}^{5}$, Peter J. Whitehouse, $\mathrm{MD}, \mathrm{PhD}^{6}$, Charmaine D.M. Royal, $\mathrm{PhD}^{7}$, \\ Thomas O. Obisesan, MD, MPH${ }^{8}$, L. Adrienne Cupples, $\mathrm{PhD}^{9}$, Melissa B. Butson, $\mathrm{ScM}^{9} \mathrm{PhD}^{6}$, \\ Grace-Ann Fasaye, ScM, CGC ${ }^{10}$, Susan Hiraki, MS, MPH ${ }^{11}$, Clara A. Chen, MA ${ }^{9}$, \\ Uwe Siebert, MD, SCD ${ }^{2,12,13}$, Robert Cook-Deegan, $\mathrm{MD}^{14}$, Robert C. Green, MD, MPH ${ }^{1,2,15,16}$ \\ for the REVEAL Study Group ${ }^{17}$
}

\begin{abstract}
Purpose: Telephone disclosure of genetic test results can improve access to services. To date, studies of its impact have focused on return of Mendelian risk information, principally hereditary cancer syndromes.

Methods: In a multisite trial of Alzheimer disease genetic risk disclosure, asymptomatic adults were randomized to receive test results in person or via telephone. Primary analyses examined patient outcomes 12 months after disclosure.

Results: Data from 257 participants showed that telephone disclosure occurred 7.4 days sooner and was 30\% shorter, on average, than in-person disclosure (both $P<0.001$ ). Anxiety and depression scores were well below cutoffs for clinical concern across protocols. Comparing telephone and in-person disclosure protocols, 99\% confidence intervals of mean differences were within noninferiority margins on scales assessing anxiety, depression, and
\end{abstract}

test-related distress, but inconclusive about positive impact. No differences were observed on measures of recall and subjective impact. Subanalyses supported noninferiority on all outcomes among apolipoprotein E $(A P O E) \varepsilon 4$-negative participants. Subanalyses were inconclusive for $A P O E \varepsilon 4$-positive participants, although mean anxiety and depression scores were still well below cutoffs for clinical concern.

Conclusion: Telephone disclosure of $A P O E$ results and risk for Alzheimer disease is generally safe and helps providers meet demands for services, even when results identify an increased risk for disease.

Genet Med advance online publication 20 July 2017

Key Words: Alzheimer disease; APOE; genetic testing; risk assessment; telephone

\section{INTRODUCTION}

Face-to-face disclosure of genetic test results has been a longstanding practice, ${ }^{1}$ but the demand for services is outpacing the capacity of most clinics. ${ }^{2,3}$ Some individuals, particularly those in rural areas, find it challenging to meet with genetic service providers in person. ${ }^{4}$ To expedite timely disclosure of genetic test results to as many patients as possible, genetic specialists are increasingly providing results via telephone. ${ }^{5-7}$

Analyses of telephone disclosure to date are encouraging. Many patients prefer it to in-person or mailed disclosure, ${ }^{8}$ and studies of its use during testing for hereditary cancer syndromes have shown comparable levels of patient understanding and satisfaction after telephone and in-person disclosure. $^{9-13}$ Two randomized trials demonstrated noninferiority of telephone disclosure on a variety of outcomes, showing that differences from in-person disclosure on scales of test-related distress, knowledge, and overall satisfaction were not clinically meaningful. ${ }^{13-15}$ To date, however, studies of telephone disclosure have almost exclusively focused on hereditary cancer syndromes.

Genetic testing to determine risk of Alzheimer disease (AD) provides a rich context for examining disclosure of genetic risk information for common, complex conditions. The $\varepsilon 4$ allele of apolipoprotein $\mathrm{E}(A P O E)$ is a prevalent and robust genetic risk factor for $\mathrm{AD},{ }^{16}$ but is neither necessary nor

${ }^{1}$ Division of Genetics, Department of Medicine, Brigham and Women's Hospital, Boston, Massachusetts, USA; ${ }^{2}$ Department of Medicine, Harvard Medical School, Boston, Massachusetts, USA; ${ }^{3}$ Departments of Internal Medicine and Human Genetics, University of Michigan, Ann Arbor, Michigan, USA; ${ }^{4}$ Department of Health Behavior and Health Education, University of Michigan School of Public Health, Ann Arbor, Michigan, USA; ${ }^{5}$ Division of Public Health Sciences, Department of Surgery, Washington University School of Medicine, St. Louis, Missouri, USA; ${ }^{6}$ Department of Neurology, Case Western Reserve University/University Hospitals, Cleveland, Ohio, USA; ${ }^{7}$ Department of African and African American Studies, Duke University, Durham, North Carolina, USA; ${ }^{8}$ Department of Medicine, Howard University College of Medicine, Washington, DC, USA;

${ }^{9}$ Departments of Biostatistics and Epidemiology, Boston University School of Public Health, Boston, Massachusetts, USA; ${ }^{10}$ National Cancer Institute, Bethesda, Maryland, USA; ${ }^{11}$ Department of Neurology, Boston University School of Medicine, Boston, Massachusetts, USA; ${ }^{12}$ UMIT-University for Health Sciences, Medical Informatics and Technology, Hall i.T., Austria; ${ }^{13}$ Harvard School of Public Health and Massachusetts General Hospital, Boston, Massachusetts, USA; ${ }^{14}$ School for the Future of Innovation in Society and Consortium for Science, Policy \& Outcomes, Arizona State University, Washington, DC, USA; ${ }^{15}$ Broad Institute of Harvard and MIT, Cambridge, Massachusetts, USA; ${ }^{16}$ Partners Personalized Medicine, Boston, Massachusetts, USA. Correspondence: Kurt D. Christensen (kchristensen@bwh.harvard.edu)

${ }^{17}$ Additional members of the REVEAL Study Group are listed after Acknowledgments. 
sufficient for disease. The Risk Evaluation and Education for Alzheimer's Disease (REVEAL) Study is a series of multicenter randomized clinical trials that have examined the impact of $\mathrm{AD}$ genetic susceptibility testing using APOE genotyping. Previous analyses have shown that testing does not increase risks for psychological harm, even among individuals who are $\varepsilon 4$-positive; ${ }^{17-19}$ and that the majority of participants accurately recall test results. ${ }^{20-22}$ Here, we report on the third REVEAL Study trial, hypothesizing that mean scores on a variety of patient outcomes would be no worse following telephone disclosure than following in-person disclosure.

\section{Study population}

\section{MATERIALS AND METHODS}

We recruited cognitively normal adults using mailings to research registries, postings on institutional research websites, referrals from collaborating physicians, and advertisements in local newspapers at study sites in Boston, MA; Cleveland, $\mathrm{OH}$; Ann Arbor, MI; and Washington, DC. We established targets to enroll equal numbers of adults over and under the age of 60 , equal numbers of men and women, and a 1:3 ratio of subjects with zero and one $\mathrm{AD}$-affected first-degree relative, respectively. Screening per prespecified criteria was conducted initially by phone, and again more extensively by study clinicians during the first in-person appointment. Individuals were excluded if they had histories suggestive of hereditary $\mathrm{AD}$ (two or more $\mathrm{AD}$-affected first-degree relatives, family members with average $\mathrm{AD}$ onset under age 60 ), or scored below an education-adjusted 87 on the Modified Mini-Mental State Examination. ${ }^{23}$ We also screened out individuals with severe anxiety or depression per validated scales (defined below).

\section{Study design}

A multidisciplinary team designed the study protocol, approved by institutional review boards at each study site and an independent external Ethics and Safety Board, and the study was registered at ClinicalTrials.gov (NCT00462917). The study protocol, statistical code, and data set are available from the authors upon request. Briefly, participants who completed a phone interview and written questionnaire received educational materials developed in a prior trial. ${ }^{17}$ Participants then met with a genetic counselor and provided a blood sample for APOE genotyping at a CLIA-certified laboratory. Participants provided informed consent by telephone prior to the initial phone interview, then again in person prior to the blood draw for genotyping. Disclosure sessions were scheduled at the discretion of individual sites. Genetic counselors disclosed APOE genotypes and AD risk information using a script that allowed participants to raise any questions or concerns they had. Numeric estimates of lifetime (cumulative incidence from birth to the age of 85 years. Range: $6 \%$ to $73 \%$ ) and remaining risk for $\mathrm{AD}$ (cumulative incidence from current age to the age of 85 years) were accompanied by graphs of $\mathrm{AD}$ risk curves. The methods for calculating risk estimates and generating risk curves were published previously. ${ }^{24,25}$ A written summary of the risk assessments were given to participants at the end of in-person disclosure sessions or mailed to participants following telephone disclosure.

A $2 \times 2$ factorial design determined whether participants received risk assessments in person or via telephone, and whether or not participants additionally learned about an association between the $\varepsilon 4$ allele of $A P O E$ and an increased risk for coronary artery disease (CAD), reported separately. ${ }^{19}$ Participants were randomized equally within strata, in blocks of size four, into "AD-only, in-person disclosure," "AD-only, telephone disclosure," "AD + CAD, in-person disclosure," and "AD + CAD, telephone disclosure" arms. Randomization strata were defined by site, age ( $<60$ vs. $\geq 60$ ), family history of $\mathrm{AD}$, and gender. Serially numbered envelopes concealed randomization statuses until the initial in-person visit. Randomization occurred prior to this appointment, which included confirmation of study eligibility, to allow consent forms customized to $\mathrm{AD}+\mathrm{CAD}$ or $\mathrm{AD}$-only randomization status to be mailed before in-person review.

Outcomes were assessed through questionnaires administered 6 weeks, 6 months, and 12 months after genotype and genetic risk disclosure. At the end of 6-week and 6-month follow-up appointments and after surveys were completed, genetic counselors verbally reminded participants about their genotypes, risk estimates, and about $A P O E-C A D$ associations (if appropriate). For safety purposes, subjects whose anxiety or depression scores exceeded standard cutoffs for severe mood disorders or increased by more than 15 points from baseline were immediately interviewed by a genetic counselor.

\section{Measures}

\section{Outcome variables}

Outcomes of interest are bolded and included anxiety, depression, test-related distress (assessed via two scales), positive impact, recall of results, and subjective impact at 6 weeks, 6 months, and 1 year after results disclosure, all assessed via self-administered questionnaires. Outcomes were selected based on concerns about how telephone disclosure may impact the communication of information. ${ }^{26}$

Psychological outcomes included measures of general anxiety, general depression, two scales assessing test-related distress, and positive impact. Anxiety was assessed using the Beck Anxiety Inventory (BAI), ${ }^{27}$ with scores ranging from 0 to 63 (>8: mild, $>15$ : moderate, $>25$ : severe). Depression was assessed using the 20-item Center for Epidemiological Studies-Depression Scale (CES-D), ${ }^{28}$ with scores ranging from 0 to 60 (>10: mild, > 16: moderate, $>26$ : severe) ${ }^{29}$ Testrelated distress was assessed with the 15-item Impact of Event Scale (IES), ${ }^{30}$ with scores ranging from 0 to $75(\geq 20$ : significant distress) and the distress subscale of Impact of Genetic Testing for Alzheimer's disease instrument (IGT-AD distress), with higher scores on a 0-60 scale indicating more negative feelings. ${ }^{31}$ Positive impact was assessed with the positive subscale of the IGT-AD, where items were reverse- 
scored such that lower scores on a 0-20 scale indicated more positive feelings. ${ }^{31}$

Recall of results was measured as the sum of correct responses when participants report back their results: (i) number of $\mathrm{AD}$ risk-increasing alleles, (ii) genotype, (iii) lifetime $\mathrm{AD}$ risk estimate, and (iv) remaining $\mathrm{AD}$ risk estimate. The risk allele and genotype items were assessed via multiple choice and included a "don't remember" option. Risk estimate items asked participants to provide their lifetime and remaining risk percentages and were considered correct if responses were within $5 \%$ of communicated results, as used in prior analyses. ${ }^{20-22}$ Item prompts encouraged participants to provide their best guess if they did not remember their estimate. An additional open-ended item asked, "What other disease did we tell you is associated with the APOE gene?" but is analyzed separately from other recall items because it was administered only to participants randomized to $A D+C A D$ disclosure.

Subjective impact was assessed by asking participants to rate the "overall impact" that the risk information had on a 5-point scale ("very negative" to "very positive").

\section{Other variables}

Participants reported demographic information during the phone interview and on the baseline written questionnaire. Personal and family history of $\mathrm{AD}$, cardiovascular disease, and other medical conditions were assessed during the blood draw appointment, as was numeracy using a validated scale with scores ranging from 0 to 8 based on the number of items a participant answers correctly. ${ }^{32}$ Participants also completed a validated 4-item version of a scale assessing self-reported comfort with numbers, with scores ranging from 1 to 6 based on mean ratings across items. ${ }^{33}$ After disclosure sessions, genetic counselors completed a chart note and indicated if topics discussed addressed any 12 issues, including the accuracy of results or preventive measures. Disclosure session length was calculated in minutes by comparing the start and finish times recorded on chart notes.

\section{Statistical analysis}

A priori goals to enroll 280 participants and achieve 256 disclosures were set to power original hypotheses to compare disclosure of $\mathrm{AD}$ and $\mathrm{CAD}$ risk information against disclosure of only $\mathrm{AD}$ risk information, the main focus of the trial. ${ }^{19}$ Here we focused on the comparison between telephone and in-person disclosure. We used $t$-tests and chi-squared tests to compare demographics of the randomization arms and to analyze who dropped out after randomization. We used chi-squared tests to compare dropout rates of study arms after randomization, but before results disclosure. We used $t$-tests of log-transformed times to compare how long after the blood draw that disclosure sessions occurred and to compare the length of disclosure sessions, and used chi-squared tests to determine whether topics discussed during disclosure sessions varied by randomization status.
Telephone disclosure was intended to streamline service delivery rather than improve patient responses. Therefore, we used a noninferiority framework to test whether outcomes were no worse after telephone disclosure than after in-person disclosure. We asserted noninferiority of telephone disclosure on measures of anxiety, depression, test-related distress, and positive impact if the upper limit for the confidence interval (CI) of mean differences between telephone and in-person randomization arms was below a predefined margin. ${ }^{34}$

We used longitudinal analyses for all outcomes, including all observed data and imputing data for the few missing observations. We used generalized linear models fit with generalized estimating equations. For analyses of BAI, CES-D, IES, IGT-AD distress, and IGT-AD positive scores, we used a log link and gamma distribution to compare outcomes by phone versus in-person randomization status, as these measures were very skewed and had a high proportion with zeros. For analyses of ordinal variables, recall was dichotomized to compare full recall (all items recalled correctly) against less than full recall, and subjective impact was dichotomized to compare responses of "very" and "somewhat positive" against responses of "neutral" and "somewhat" or "very negative." Analyses of recall and subjective impact used a logit link and binomial distribution. We used an autoregressive working correlation structure with robust standard errors to account for the repeated measures within participant. A value of one was added to BAI, CES-D, IES, and IGT measures to shift their distributions away from zero. Models included terms for phone or in-person disclosure randomization status, time as a categorical variable, interaction between time and randomization arm, and the corresponding baseline psychological measure where applicable. Analyses on the IGT-AD distress subscale included a term to account for an interaction between telephone/ in-person randomization status and $\mathrm{AD}$-only/AD $+\mathrm{CAD}$ randomization status $(P<0.05)$. Additional analyses were conducted to compare arms in APOE \&4-positive and APOE $\varepsilon 4$-negative participants, as well as to further adjust for age, gender, education, race, family history of $\mathrm{AD}, \mathrm{AD}+\mathrm{CAD}$ or $\mathrm{AD}$-only information disclosure, numeracy, and self-reported comfort in analyses of information recall. ${ }^{20}$ We also conducted analyses where missing data were not imputed to minimize potential biases in noninferiority analyses. ${ }^{34}$ These adjusted analyses and available case analyses are omitted from this report, because unadjusted models using imputed data were considered conservative in comparison (i.e., we report noninferiority only when it was demonstrated in all analyses). We used contrasts to compare randomization arms at specific time points and overall for a time-averaged comparison.

Consistent with analyses of prior REVEAL Study trials, ${ }^{17,19}$ outcomes at 12 months were considered primary, while outcomes at 6 months and 6 weeks were considered secondary. Data for $\mathrm{AD}+\mathrm{CAD}$ and $\mathrm{AD}$-only disclosure arms were pooled in analyses because interactions between $\mathrm{AD}$-only/AD + $\mathrm{CAD}$ randomization status and in-person/ telephone disclosure randomization status were not observed 
( $P$ values for two-way tests of interaction between the two treatment arms and three-way interactions between the treatment arms and time were all greater than 0.05 except for analyses of IGT-AD distress scores, as noted earlier). The margin of noninferiority for BAI, CES-D, and IES scores was 5 points, as used in prior REVEAL Study trials, indicative of medium to large effect sizes (Cohen's $d=1.03,0.64$, and 0.66, respectively). ${ }^{17,18}$ This criterion was a more conservative margin than 5- to 10 -point definitions of "clinically meaningful" differences used in other studies that are based on the intervals between cutoffs for minimal, mild, moderate, and severe anxiety and depression. ${ }^{35-37}$ For IGT-AD scores where prior studies have not identified a meaningful difference, we set margins following the strategy used in a related noninferiority analysis: ${ }^{13}$ three points, representing a shift from "sometimes" to "never" on a single item and indicative of medium effect sizes (Cohen's $d=0.43$ and 0.48 for distress and positive subscales, respectively). Noninferiority margins were not established for recall or subjective impact measures.

We used 99\% CIs for primary analyses (12-month outcomes) to account for testing of seven outcomes (anxiety, depression, test-related distress per the IES and IGT-AD, positive impact, and recall of results). These CIs are more conservative than a Bonferroni correction, given guidelines to use one-sided tests. ${ }^{34}$ To be consistent across outcomes, we used 99\% CIs on all secondary analyses. Additional secondary analyses used 99\% CIs of two-group tests of proportions to compare recall rates on individual recall items by randomization status and $\mathrm{McNemar}$ tests to compare whether participants were more likely to recall specific items more often than others.

Analyses included only participants receiving genetic risk information (genotype data for participants who provided blood but dropped out of the study before the disclosure session was destroyed per the institutional review boardapproved protocol). We assumed data were missing at random and imputed missing values using multiple imputation (Markov chain Monte Carlo procedures with 40 imputed data sets). All analyses were conducted using SAS, version 9.3 (SAS Institute, Cary, NC).

\section{RESULTS}

Of 290 randomized participants, 257 (88.6\%) received genetic risk disclosure (Figure 1) with no observed differences in dropout rates between telephone and in-person disclosure arms $(\Delta=0.1 \%, P=0.99)$. Twenty-four randomized participants withdrew before disclosure for the following nonexclusive reasons: concerns about potential emotional responses (8); study demands (4); personal or family health problems (4); concerns about privacy, confidentiality, or discrimination (4); lack of AD prevention options (3); moved (2); no longer interested (1); concerns about test limitations (1); and desire for higher remuneration (1). One participant passed away, and four were lost to follow-up. Four others did not meet eligibility criteria due to low Modified Mini-Mental State scores, high CES-D scores, questionable family history of $\mathrm{AD}$, and failure to attend study appointments.
Demographic characteristics of participants who received genetic risk information did not vary by disclosure method (Table 1) and were similar to those of the second REVEAL Study trial except for the inclusion of individuals without an $\mathrm{AD}$-affected first-degree relative. ${ }^{17}$ On average, telephone disclosure occurred 7.4 days sooner than in-person disclosure (27.8 vs. 35.2 days after the blood draw, respectively; $P=0.002$ ). Disclosure sessions ranged in duration from 6 to 40 minutes for telephone disclosure and 5 to 50 minutes for in-person disclosure, and, on average, telephone disclosure sessions were 6.6 minutes shorter than in-person disclosure sessions $(P<0.001)$. Disclosure session chart notes showed that participants were more likely to discuss preventive options during in-person disclosure than telephone disclosure (26\% vs. $14 \%$, respectively, $P=0.015)$. Disclosure sessions did not vary in length by $A P O E$ status $(P=0.15$ in bivariate analyses, $P=0.16$ in analyses controlling for telephone/ in-person randomization status).

One year after disclosure and across randomization arms, participants remembered 3.0 of 4 items correctly, on average, and scored well below cutoffs for clinical concern on anxiety and depression scales (see Table 2). Overall, $24 \%$ of participants receiving in-person disclosure and $23 \%$ of participants receiving telephone disclosure reported moderate anxiety, depression, or test-related distress at one or more follow-up time points, with no differences observed by disclosure method $(P=0.61)$. The majority of participants $(65.6 \%)$ rated the subjective impact of their risk assessment as positive, while $7.9 \%$ rated it negative and $26.5 \%$ rated it neutral. Mean scores on measures of general anxiety and depression were well below cutoffs for clinical concern at all time points, and positive impact scores increased over time $(P<0.001)$. With the exception of IGT-AD positive scores, CIs for mean differences between telephone and in-person disclosure arms were below prespecified margins of noninferiority for all scales at all time points in analyses that were not stratified by $A P O E$ status (Table 2) and in analyses of $\varepsilon 4$ negative participants (Table 3). However, analyses in $\varepsilon 4$ positive participants supported noninferiority of telephone disclosure at 12 months only on anxiety. Furthermore, upper limits of 99\% CIs exceed margins for noninferiority on IES and IGT-AD distress scores at the 6-week and 6-month time points, and upper limits of $99 \%$ CIs exceeded margins for noninferiority on the IGT-AD positive scale at 6 weeks.

Additional analyses suggested that telephone disclosure outperformed in-person disclosure on some components of recalling results (Table 4). Participants were more likely to correctly recall lifetime $\mathrm{AD}$ risk estimates at 6 months ( $\Delta=17.6 \%, 99 \% \mathrm{CI}: 4.6 \%$ to $30.6 \%)$ and 12 months ( $\Delta=15.8 \%, 99 \%$ CI: $2.1 \%$ to $29.6 \%$ ) after telephone disclosure compared to in-person disclosure. Independent of disclosure method or genotype, recall scores were higher at 6 weeks than at 6 months $(\Delta=0.3, P<0.001)$ or 12 months ( $\Delta=0.4, P<0.001)$. At 12 months, $\varepsilon 4$-positive participants were more likely than $\varepsilon 4$-negative participants to correctly recall their genotype $(74.0 \%$ vs. $56.3 \%, P=0.008)$. Across 


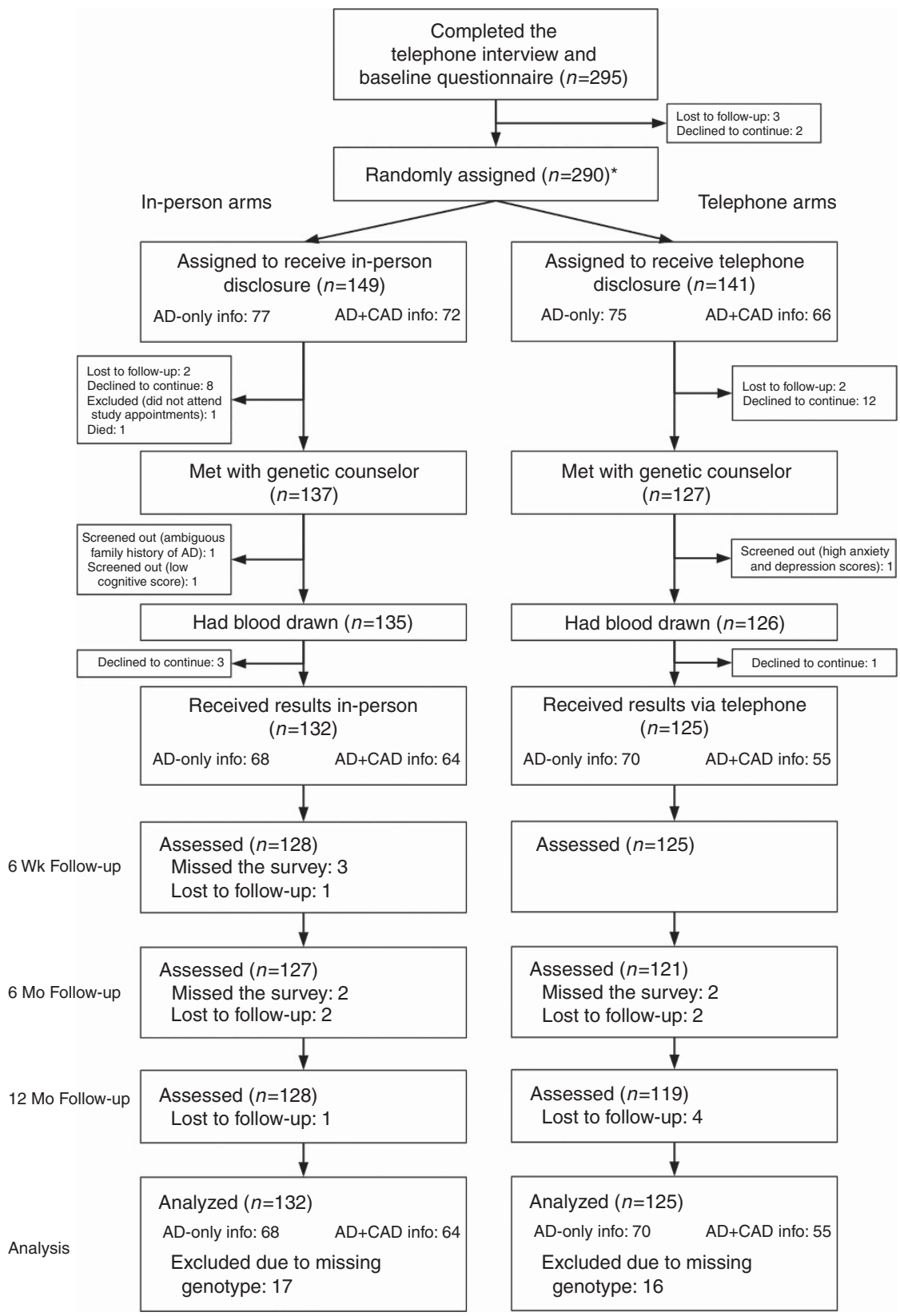

Figure 1 Enrollment flow chart. *A second randomization occurred at this point and is described in "Materials and Methods." AD, Alzheimer disease; $C A D$, coronary artery disease.

genotypes and randomization arms, data for individual recall items were highly correlated $(r>0.57$ in all pairwise comparisons), although participants were less likely to recall their specific genotypes compared to the number of risk alleles they had or their lifetime or remaining $\mathrm{AD}$ risk estimates (all pairwise comparisons $P<0.001$ ). Participants who did not accurately recall numeric AD risk estimates tended to provide estimates lower than what were reported during disclosure sessions: $64.2 \%$ of participants with inaccurate lifetime risk recall provided estimates lower than those reported, and $61.5 \%$ of participants with inaccurate remaining risk recall provided estimates lower than those reported.

\section{DISCUSSION}

On average, telephone disclosure of genetic risk information about $\mathrm{AD}$ was safe and did not increase psychological risks. Although we were unable to demonstrate statistical noninferiority for some outcomes within the subset of individuals who learned that they were $A P O E$ \&4-positive, their mean anxiety and depression scores were still well below cutoffs for clinical concern. These findings are notable because risk 
Table 1 Characteristics of subjects who received genetic risk disclosure

\begin{tabular}{|c|c|c|c|}
\hline \multirow[b]{2}{*}{ Characteristic } & \multicolumn{2}{|c|}{ Randomization arm } & \multirow[b]{2}{*}{$P$} \\
\hline & $\begin{array}{l}\text { In person } \\
(n=132)\end{array}$ & $\begin{array}{l}\text { Telephone } \\
(n=125)\end{array}$ & \\
\hline \multicolumn{4}{|l|}{ Age: yr } \\
\hline Mean \pm SD & $58.2 \pm 12.9$ & $58.1 \pm 13.0$ & 0.95 \\
\hline Range & 21-83 & $22-82$ & \\
\hline Female sex: $n(\%)$ & $73(55)$ & $68(54)$ & 0.88 \\
\hline African-American race: $n(\%)$ & $21(16)$ & $17(14)$ & 0.60 \\
\hline \multicolumn{4}{|l|}{ Education: yr } \\
\hline Mean $\pm S D$ & $16.6 \pm 2.4$ & $17.0 \pm 2.2$ & 0.18 \\
\hline Range & $10-20$ & $12-20$ & \\
\hline Currently married: $n(\%)$ & $78(59)$ & $75(60)$ & 0.88 \\
\hline Site: $n(\%)$ & & & 0.99 \\
\hline Boston, MA & $40(30)$ & $38(30)$ & \\
\hline Cleveland, $\mathrm{OH}$ & $32(25)$ & $32(25)$ & \\
\hline Ann Arbor, Ml & $35(27)$ & $33(26)$ & \\
\hline Washington, DC & $25(19)$ & $22(18)$ & \\
\hline Parent or sibling with AD: $n$ (\%) & $89(67)$ & $89(71)$ & 0.51 \\
\hline Pleiotropic disclosure: $n(\%)$ & $64(48)$ & $55(44)$ & 0.47 \\
\hline Has $\varepsilon 4$ allele: $n(\%)$ & & & 0.86 \\
\hline One copy & $37(28)$ & $34(27)$ & \\
\hline Two copies & $7(5)$ & $5(4)$ & \\
\hline Numeracy: mean \pm SD & $7.1 \pm 1.3$ & $7.4 \pm 1.3$ & 0.06 \\
\hline $\begin{array}{l}\text { Comfort with numbers: } \\
\text { mean } \pm S D\end{array}$ & $4.5 \pm 1.0$ & $4.7 \pm 0.9$ & 0.10 \\
\hline BAl score: mean \pm SD & $3.4 \pm 3.5$ & $3.6 \pm 3.5$ & 0.55 \\
\hline CES-D score: mean \pm SD & $5.9 \pm 5.0$ & $5.5 \pm 5.2$ & 0.56 \\
\hline
\end{tabular}

AD, Alzheimer disease; BAl, Beck Anxiety Inventory; CES-D, Center for Epidemiological Studies-Depression Scale.

disclosure for $\mathrm{AD}$ has been used as an example of potentially distressing information, given the lack of proven preventive strategies. Anticipation of telephone disclosure did not appear to affect participants' willingness to receive a genetic risk assessment, and wait times for disclosure sessions were shorter when results were disclosed by telephone, with the disclosure sessions themselves briefer than in-person disclosure sessions.

These findings are encouraging given that many genetic service providers are already disclosing test results via phone. Telephone disclosure is a longstanding practice in prenatal settings given time-sensitive implications for pregnancies, and it is now in wider use in other settings when results suggest no carrier or disease risks. ${ }^{6}$ Our data are consistent with findings from research on hereditary breast and ovarian cancer syndromes, showing that telephone disclosure does not increase risks for misremembering results or psychological harms. ${ }^{11-13}$ In fact, telephone disclosure seemed to improve participants' abilities to retain numeric risk estimates. This improvement may be because participants received information at two distinct time points, first during phone disclosure sessions, then again when participants received the information via mail. The time savings noted in our study were also
Table 2 Mean outcome scores by randomization group and time after $A P O E$ genotype disclosure

\begin{tabular}{lccc} 
Variable & $\begin{array}{c}\text { In person } \\
(\boldsymbol{n}=\mathbf{1 3 2})\end{array}$ & $\begin{array}{c}\text { Phone } \\
(\boldsymbol{n}=\mathbf{1 2 5})\end{array}$ & $\begin{array}{c}\text { Difference } \\
(\mathbf{9 9 \%} \mathrm{Cl})\end{array}$ \\
\hline 12-mo outcomes & & & \\
\hline BAI & 3.6 & 3.5 & $-0.2(-1.5$ to 1.2$)$ \\
\hline CES-D & 6.2 & 7.4 & $1.1(-1.2$ to 3.4$)$ \\
\hline IES & 3.1 & 3.7 & $0.5(-2.1$ to 3.1$)$ \\
\hline IGT distress & 4.4 & 4.2 & $-0.3(-2.7$ to 2.2$)$ \\
\hline IGT positive & 13.1 & 14.3 & $1.2(-1.3$ to 3.8$)$ \\
\hline Full recall & $37.3 \%$ & $50.1 \%$ & $1.7(0.8 \text { to } 3.4)^{\mathrm{a}}$ \\
\hline Positive & $68.7 \%$ & $63.8 \%$ & $0.8(0.4 \text { to } 1.7)^{\mathrm{a}}$
\end{tabular}

subjective impact

6-mo outcomes

$\begin{array}{lccc}\text { BAI } & 3.1 & 2.8 & -0.3(-1.4 \text { to } 0.9) \\ \text { CES-D } & 5.3 & 6.1 & 0.8(-1.2 \text { to } 2.8) \\ \text { IES } & 3.8 & 4.0 & 0.2(-2.4 \text { to } 2.7) \\ \text { IGT distress } & 4.1 & 4.6 & 0.5(-1.7 \text { to } 2.7) \\ \text { IGT positive } & 11.3 & 11.7 & 0.4(-2.0 \text { to } 2.8) \\ \text { Full recall } & 46.6 \% & 55.4 \% & 1.4(0.7 \text { to } 2.8)^{\mathrm{a}} \\ \text { Positive } & 71.8 \% & 64.7 \% & 0.7(0.4 \text { to } 1.5)^{\mathrm{a}} \\ \text { subjective impact } & & & \end{array}$

6-wk outcomes

$\begin{array}{lccc}\text { BAI } & 2.9 & 3.1 & 0.3(-0.7 \text { to } 1.3) \\ \text { CES-D } & 4.8 & 6.5 & 1.7(-0.2 \text { to } 3.6) \\ \text { IES } & 3.9 & 4.4 & 0.5(-2.1 \text { to } 3.1) \\ \text { IGT distress } & 4.6 & 5.1 & 0.5(-1.8 \text { to } 2.7) \\ \text { IGT positive } & 9.2 & 10.4 & 1.1(-1.1 \text { to } 3.4) \\ \text { Full recall } & 61.6 \% & 61.9 \% & 1.0(0.5 \text { to } 2.0)^{\mathrm{a}} \\ \text { Positive } & 63.9 \% & 56.8 \% & 0.7(0.4 \text { to } 1.5)^{\mathrm{a}}\end{array}$

subjective impact

Time-averaged outcomes

\begin{tabular}{lccl} 
BAI & 3.2 & 3.1 & $0.0(-0.9$ to 0.9$)$ \\
CES-D & 5.4 & 6.6 & $1.0(-0.4$ to 2.8$)$ \\
IES & 3.6 & 4.0 & $0.4(-1.9$ to 2.7$)$ \\
IGT distress & 4.4 & 4.6 & $0.2(-1.9$ to 2.3$)$ \\
IGT positive & 11.1 & 12.0 & $0.9(-1.1$ to 3.0$)$ \\
Full recall & $44.4 \%$ & $52.4 \%$ & $1.4(0.8 \text { to } 2.4)^{\mathrm{a}}$ \\
Positive & $65.5 \%$ & $58.3 \%$ & $0.8(0.4 \text { to } 1.3)^{\mathrm{a}}$ \\
subjective impact & & & \\
\hline
\end{tabular}

BAI, Beck Anxiety Inventory; CES-D, Center for Epidemiological Studies-Depression Scale; $\mathrm{Cl}$, confidence interval; IES, Impact of Event Scale; IGT, Impact of Genetic Testing for Alzheimer's disease instrument.

Scores were estimated using generalized estimating equations with log link and $\gamma$ distribution for continuous measures and with logit link and binomial distribution for dichotomized measures, with adjustment for corresponding baseline values (where applicable) and the genetic counselor providing disclosure.

aDifferences for dichotomized outcomes, full recall, and positive subjective impact, represent odds ratios (i.e., the odds of full recall or positive subjective impact following telephone disclosure compared to in-person disclosure).

comparable to the time savings observed in the BRCA1/2 telephone disclosure studies, although our overall session lengths in our trial were often shorter. The comparably greater time needed for disclosing BRCA1/2 test results may be attributable to time needed to discuss medical management decisions and implications for other family members. 
Table 3 Mean outcome scores, stratified by $A P O E$ status

\begin{tabular}{|c|c|c|c|c|c|c|}
\hline & \multicolumn{3}{|c|}{$A P O E$ \&4 noncarriers } & \multicolumn{3}{|c|}{$A P O E$ \& 4 carriers } \\
\hline \multicolumn{7}{|l|}{ 12-mo outcomes } \\
\hline CES-D & 6.0 & 6.5 & $0.6(-1.8$ to 2.9$)$ & 6.8 & 9.2 & $2.4(-2.6$ to 7.4$)$ \\
\hline IES & 2.8 & 2.0 & $-0.8(-3.0$ to 1.4$)$ & 3.7 & 7.1 & $3.4(-2.8$ to 9.7$)$ \\
\hline IGT distress & 3.8 & 3.1 & $-0.7(-3.3$ to 1.8$)$ & 5.6 & 6.4 & $0.8(-4.2$ to 5.8$)$ \\
\hline Full recall & $28.6 \%$ & $44.4 \%$ & $2.1(0.9 \text { to } 5.0)^{a}$ & $47.4 \%$ & $52.4 \%$ & $1.2(0.4 \text { to } 3.7)^{a}$ \\
\hline Positive subjective impact & $76.1 \%$ & $72.2 \%$ & $0.8(0.3 \text { to } 2.0)^{\mathrm{a}}$ & $47.4 \%$ & $37.5 \%$ & $0.6(0.2 \text { to } 2.2)^{\mathrm{a}}$ \\
\hline \multicolumn{7}{|l|}{ 6-mo outcomes } \\
\hline BAl & 3.2 & 2.5 & $-0.6(-1.9$ to 0.6$)$ & 2.9 & 3.4 & $0.5(-1.8$ to 2.7$)$ \\
\hline CES-D & 5.1 & 5.9 & $0.8(-1.4$ to 3.0$)$ & 5.7 & 6.6 & $0.9(-3.0$ to 4.8$)$ \\
\hline Positive subjective impact & $81.5 \%$ & $76.2 \%$ & $0.7(0.3 \text { to } 2.0)^{\mathrm{a}}$ & $44.4 \%$ & $33.3 \%$ & $0.6(0.2 \text { to } 2.2)^{a}$ \\
\hline \multicolumn{7}{|l|}{ 6-wk outcomes } \\
\hline BAl & 2.8 & 2.9 & $0.0(-1.2$ to 1.2$)$ & 2.9 & 3.8 & $0.9(-0.9$ to 2.6$)$ \\
\hline CES-D & 4.9 & 6.7 & $1.8(-0.6$ to 3.0$)$ & 4.4 & 5.9 & $1.6(-1.3$ to 4.4$)$ \\
\hline IES & 2.7 & 3.3 & $0.6(-1.8$ to 3.0$)$ & 6.3 & 6.6 & $0.3(-5.4$ to 6.0$)$ \\
\hline IGT distress & 3.8 & 4.0 & $0.3(-2.0$ to 2.5$)$ & 6.4 & 7.3 & $0.9(-3.8$ to 5.6$)$ \\
\hline IGT positive & 7.7 & 8.8 & $1.1(-1.4$ to 3.5$)$ & 12.1 & 13.8 & $1.6(-2.0$ to 5.2$)$ \\
\hline Full recall & $60.0 \%$ & $63.0 \%$ & $1.1(0.5 \text { to } 2.6)^{\mathrm{a}}$ & $50.0 \%$ & $50.0 \%$ & $1.0(0.3 \text { to } 3.1)^{\mathrm{a}}$ \\
\hline Positive subjective impact & $73.0 \%$ & $68.8 \%$ & $0.8(0.3 \text { to } 2.0)^{\mathrm{a}}$ & $41.2 \%$ & $23.1 \%$ & $0.4(0.1 \text { to } 1.5)^{\mathrm{a}}$ \\
\hline \multicolumn{7}{|l|}{ Time-averaged outcomes } \\
\hline Positive subjective impact & $76.7 \%$ & $72.2 \%$ & $0.8(0.4 \text { to } 1.7)^{\mathrm{a}}$ & $44.4 \%$ & $33.3 \%$ & $0.6(0.2 \text { to } 1.5)^{a}$ \\
\hline
\end{tabular}

BAl, Beck Anxiety Inventory; CES-D,Center for Epidemiological Studies-Depression Scale; Cl, confidence interval; IES,Impact of Event Scale; IGT, Impact of Genetic Testing for Alzheimer's disease instrument.

Scores were estimated using generalized estimating equations with log link and $\gamma$ distribution for continuous measures and with logit link and binomial distribution for dichotomized measures, with adjustment for corresponding baseline values and the genetic counselor providing disclosure.

a Differences for dichotomized outcomes, full recall, and positive subjective impact, represent odds ratios (i.e., the odds of full recall or positive subjective impact following telephone disclosure compared to in-person disclosure).

Genotype-specific analyses of our data suggest that genetic service providers should be mindful of the potential for additional distress when disclosing results indicating increased risk via telephone. Noninferiority was supported on all psychological outcomes except positive impact at 12 months among $\varepsilon 4$-negative participants. However, noninferiority of telephone disclosure was not demonstrated on some 12-month outcomes among ع4-positive participants. Possible explanations for this include the reduced ability of providers to read nonverbal cues since genetic counselors are trained to use and respond to body language to encourage patients to engage with information and to reduce discomfort. ${ }^{1}$ Telephone conversations are often more succinct than those that are face to face, ${ }^{38}$ and participants or genetic counselors in our study may have felt pressure to be parsimonious about what they addressed on the phone. Our telephone disclosure protocol did not allow participants to view reports while genetic counselors were discussing results, and made it difficult for participants to have a support person present during disclosure. Whatever the reasons, genetic service providers may need to be selective about when to disclose potentially distressing genetic risk information via telephone. Predisclosure anxiety and depression scores are the strongest predictors of postdisclosure outcomes. Therefore, telephone disclosure of $\varepsilon 4$-positive results to individuals with significant anxiety or depression may not be optimal. ${ }^{18}$ One 
Table 4 Percentages recalling specific genetic test results correctly by disclosure method and time point, adjusted for APOE genotype

\begin{tabular}{|c|c|c|c|}
\hline Information & In person $(n=132)$ & Telephone $(n=125)$ & Difference $(99 \% \mathrm{Cl})$ \\
\hline \multicolumn{4}{|l|}{1 Year } \\
\hline Number of risk alleles & $80.5 \%$ & $79.8 \%$ & $-0.7 \%(-13.5 \%$ to $12.2 \%)$ \\
\hline Presence/absence of a risk allele & $81.9 \%$ & $83.8 \%$ & $1.9 \%(-10.2 \%$ to $14.0 \%)$ \\
\hline Lifetime $A D$ risk estimate $( \pm 5 \%)$ & $66.4 \%$ & $82.2 \%$ & $15.8 \%(2.1 \%$ to $29.6 \%)$ \\
\hline Remaining $A D$ risk estimate $( \pm 5 \%)$ & $71.6 \%$ & $83.6 \%$ & $12.0 \%(-1.2 \%$ to $25.3 \%)$ \\
\hline Number of risk alleles & $85.8 \%$ & $83.8 \%$ & $-2.0 \%(-13.5 \%$ to $9.6 \%)$ \\
\hline Presence/absence of a risk allele & $86.8 \%$ & $85.6 \%$ & $-1.2 \%(-12.3 \%$ to $9.9 \%)$ \\
\hline Genotype & $61.4 \%$ & $66.0 \%$ & $4.6 \%(-10.8 \%$ to $20.1 \%)$ \\
\hline Lifetime $A D$ risk estimate $( \pm 5 \%)$ & $69.1 \%$ & $86.7 \%$ & $17.6 \%(4.6 \%$ to $30.6 \%)$ \\
\hline Remaining $A D$ risk estimate $( \pm 5 \%)$ & $77.0 \%$ & $80.3 \%$ & $3.3 \%(-9.9 \%$ to $16.4 \%)$ \\
\hline Presence/absence of a risk allele & $90.3 \%$ & $86.4 \%$ & $-3.9 \%(-14.2 \%$ to $6.4 \%)$ \\
\hline Genotype & $70.9 \%$ & $79.2 \%$ & $8.3 \%(-5.5 \%$ to $22.2 \%)$ \\
\hline Lifetime $A D$ risk estimate $( \pm 5 \%)$ & $82.9 \%$ & $86.0 \%$ & $3.2 \%(-8.5 \%$ to $14.8 \%)$ \\
\hline Remaining $A D$ risk estimate $( \pm 5 \%)$ & $86.3 \%$ & $91.9 \%$ & $5.6 \%(-4.3 \%$ to $15.6 \%)$ \\
\hline Additional disease association ${ }^{a}$ & $78.1 \%$ & $83.6 \%$ & $5.5 \%(-13.0 \%$ to $24.0 \%)$ \\
\hline
\end{tabular}

$A D$, Alzheimer disease; $C A D$, coronary artery disease; $C l$, confidence interval.

a Item was administered to participants randomized to $A D+C A D$ disclosure, only.

strategy that many clinics have already implemented is to have an in-person consultation following telephone disclosure when patients have an increased genetic risk. ${ }^{10,11}$

Our study also evaluated recall of genetic test results one year after disclosure. Typically, studies have examined recall immediately after or within 1-2 months of disclosure. Even after reiterating information 6 weeks and 6 months after disclosure, we observed a steady decrease in recall over time. Of note, this trial had higher percentages of correct recall, even at 6 weeks, than the first REVEAL Study trial, ${ }^{21}$ perhaps due to the study population (e.g., more self-referred participants, individuals without $\mathrm{AD}$-affected first-degree relatives) or due to modifications in disclosure protocols. Data from this trial also reinforced findings from prior trials, showing that individuals who did not recall their lifetime and remaining risks correctly tended to underestimate their risk estimates, and that $\varepsilon 4$-positive individuals were more likely to correctly recall their genotype than $\varepsilon 4$-negative individuals. ${ }^{21}$ It is likely that individuals who were APOE ع4-positive saw a greater need to remember this information given their increased risk status.

\section{Limitations}

Study participants had higher education and numeracy than the general population. Measures were self-administered, and we could not ascertain whether participants reviewed written summaries during recall assessments. For questions about lifetime and remaining $\mathrm{AD}$ risk estimates, participants were not given a "don't remember" option, and participants may have been categorized as correctly recalling information after guessing. Results may not generalize to situations in which results might be disclosed by health-care providers without genetics expertise. Post hoc calculations based on the differences that we observed suggested that we only had approximately $17 \%$ power to confirm noninferiority among ع4-positive participants on the IES, where differences were closest to noninferiority margins, and that we would need approximately $106 \varepsilon 4$-positive participants in each arm to achieve $80 \%$ power. Our sample size also limited our ability to examine interactions between telephone disclosure and factors such as education and race. ${ }^{9}$ Our study did not assess potential benefits such as increasing access to testing, decreasing costs to patients, or opening up clinic slots.., 9 We also did not assess the impact of telephone disclosure on outcomes such as risk perceptions, health behaviors, and advance planning, although we have reported on the impact of genetic risk disclosure for $\mathrm{AD}$ on these outcomes previously. ${ }^{19,39-41}$ Such analyses may be important given differences we observed between randomization arms in the likelihood of discussing prevention during disclosure sessions.

\section{Conclusion}

There remain important challenges to telephone disclosure of genetic test results, including reimbursement policies that 
encourage in-person visits, but payers are increasingly willing to pay for telephone consultations, ${ }^{42}$ and companies are now offering telephone genetic counseling. In addition, efforts are already under way to improve the timely disclosure of test results using patient portals associated with electronic medical record systems, mailing negative results, Web-based disclosure, and video conferencing. AD risk disclosure is likely to grow in importance as prediction algorithms improve by incorporating polygenic, clinical, and environmental risk factors. ${ }^{43}$ Our results regarding telephone disclosure are encouraging given the need for efficient and effective approaches for conveying risk information and test results for common, complex diseases.

\section{ACKNOWLEDGMENTS}

Work was supported by National Institutes of Health grants HG002213, HG006500, HG009173, HD077671, HG009173, AG013846, RR000533, RR010284, AG053760, and TR001102.

\section{Additional REVEAL Study Group members}

G. Annas, Boston University School of Medicine, Boston; D. Blacker, Harvard School of Public Health, Boston; C. Chen, Boston University School of Public Health, Boston; E. Cox, Weill Cornell Medical College, New York; J.G. Davis, Weill Cornell Medical College, New York; L. Farrer, Boston University School of Medicine and Boston University School of Public Health, Boston; P. Griffith, Morehouse School of Medicine, Atlanta; K. Harkins, Perelman School of Medicine, Philadelphia; M. Johnson, Howard University, Washington, DC; S. Johnson, Howard University, Washington, DC; E. Juengst, University of North Carolina School of Medicine, Chapel Hill; J. Karlawish, Perelman School of Medicine, Philadelphia; L. Le, University of Michigan School of Public Health, Ann Arbor; E. Levison, Division of Genetics, New York Presbyterian Hospital; E, McCarty Wood, Perelman School of Medicine, Philadelphia; T. Obisesan, Howard University, Washington, DC; D. Perry, Illumina, San Diego; R. Petersen, Mayo Alzheimer's Disease Research Center, Rochester; S. Post, Stony Brook University, Stony Brook; K. Quaid, Indiana University School of Medicine, Indianapolis; L. Ravdin, Weill Cornell Medical College, New York; N. Relkin, Weill Medical College of Cornell University, New York; D. Roter, Johns Hopkins Bloomberg School of Public Health, Baltimore; A. Sadovnick, University of British Columbia, Vancouver; S. Sami, Case Western Reserve University, Cleveland; P. Sankar, Perelman School of Medicine, Philadelphia; R. Stern, Boston University School of Medicine, Boston; E. Topol, Scripps Research Institute, La Jolla; N. Wang, Boston University School of Public Health, Boston; L. Waterston, Maine Medical Center, Portland; L. Wright, Medical College of Georgia, Athens.

\section{DISCLOSURE}

R.C.G. is compensated for speaking or advisory services from AIA, GenePeeks, Helix, Illumina, Prudential, Ohana, and Veritas, and is cofounder of and adviser to Genome Medical, Inc. The other authors declare no conflicts of interest.

\section{REFERENCES}

1. Uhlmann WR, Schuette JL, Yashar BM. A Guide to Genetic Counseling, 2nd edn, Wiley-Blackwell: Hoboken, NJ, 2009.

2. Taylor MRG, Edwards JG, Ku L. Lost in transition: challenges in the expanding field of adult genetics. Am J Med Genet C Semin Med Genet. 2006;142C:294-303.

3. Carroll J. Genetic testing: counselors desperately needed. Biotechnol Healthc. 2009;6:14-22.

4. Koil CE, Everett JN, Hoechstetter L, Ricer RE, Huelsman KM. Differences in physician referral practices and attitudes regarding hereditary breast cancer by clinical practice location. Genet Med. 2003;5:364-369.

5. Bradbury AR, Patrick-Miller L, Fetzer D, et al. Genetic counselor opinions of, and experiences with telephone communication of BRCA1/2 test results. Clin Genet. 2011;79:125-131.

6. Wham D, Vu T, Chan-Smutko G, Kobelka C, Urbauer D, Heald B. Assessment of clinical practices among cancer genetic counselors. Fam Cancer. 2010;9:459-468.

7. Baumanis L, Evans J, Callanan N, Susswein L. Telephoned BRCA1/2 genetic test results: prevalence, practice, and patient satisfaction. J Genet Couns. 2009;18:447-463.

8. O'Shea R, Meany M, Carroll C, et al. Predictive genetic testing and alternatives to face to face results disclosure: a retrospective review of patients preference for alternative modes of BRCA 1 and 2 results disclosure in the Republic of Ireland. J Genet Couns. 2016:25:422-431.

9. Patrick-Miller L, Egleston BL, Daly M, et al. Implementation and outcomes of telephone disclosure of clinical BRCA1/2 test results. Patient Educ Couns. 2013;93:413-419.

10. Klemp JR, O'Dea A, Chamberlain C, Fabian CJ. Patient satisfaction of BRCA1/2 genetic testing by women at high risk for breast cancer participating in a prevention trial. Fam Cancer. 2005:4:279-284.

11. Doughty Rice C, Ruschman J, Martin L, Manders J, Miller E. Retrospective comparison of patient outcomes after in-person and telephone results disclosure counseling for BRCA1/2 genetic testing. Fam Cancer. 2010;9: 203-212.

12. Jenkins J, Calzone K, Dimond E, et al. Randomized comparison of phone versus in-person BRCA1/2 predisposition genetic test result disclosure counseling. Genet Med. 2007:9:487-495.

13. Schwartz MD, Valdimarsdottir HB, Peshkin BN, et al. Randomized noninferiority trial of telephone versus in-person genetic counseling for hereditary breast and ovarian cancer. J Clin Oncol. 2014;32:618-626.

14. Kinney AY, Butler KM, Schwartz MD, et al. Expanding access to BRCA1/2 genetic counseling with telephone delivery: a cluster randomized trial. J Natl Cancer Inst. 2014;106:dju328.

15. Kinney $A Y$, Steffen $L E$, Brumbach $B H$, et al. Randomized noninferiority trial of telephone delivery of BRCA1/2 genetic counseling compared with in-person counseling: 1-year follow-up. J Clin Oncol. 2016;34:2914-2924.

16. Farrer LA, Cupples LA, Haines JL, et al. Effects of age, sex and ethnicity on the association between apolipoprotein $\mathrm{E}$ genotype and Alzheimer disease: a meta-analysis. JAMA. 1997;278:1349-1356.

17. Green RC, Christensen KD, Cupples LA, et al. A randomized noninferiority trial of condensed protocols for genetic risk disclosure of Alzheimer's disease. Alzheimers Dement. 2015;11:1222-1230.

18. Green RC, Roberts JS, Cupples LA, et al. Disclosure of APOE genotype for risk of Alzheimer's disease. N Engl J Med. 2009;361:245-254.

19. Christensen KD, Roberts JS, Whitehouse PJ, et al. Disclosing pleiotropic effects during genetic risk assessment for Alzheimer disease. A randomized trial. Ann Intern Med. 2016;164:155-163.

20. Besser AG, Sanderson SC, Roberts JS, et al. Factors affecting recall of different types of personal genetic information about Alzheimer's disease risk: the REVEAL Study. Public Health Genomics. 2015;18:78-86.

21. Eckert SL, Katzen H, Roberts JS, et al. Recall of disclosed apolipoprotein E genotype and lifetime risk estimate for Alzheimer's disease: the REVEAL Study. Genet Med. 2006;8:746-751.

22. Roberts JS, Chen CA, Uhlmann WR, Green RC. Effectiveness of a condensed protocol for disclosing APOE genotype and providing risk education for Alzheimer disease. Genet Med. 2012;14:742-748.

23. Teng EL, Chui HC. The Modified Mini-Mental State (3MS) Examination. J Clin Psychiatry. 1987;48:314-318.

24. Christensen KD, Roberts JS, Royal CDM, et al. Incorporating ethnicity into genetic risk assessment for Alzheimer disease: the REVEAL Study experience. Genet Med. 2008:10:207-214.

25. Cupples LA, Farrer LA, Sadovnick AD, Relkin N, Whitehouse $P$, Green RC. Estimating risk curves for first-degree relatives of patients with Alzheimer's disease: the REVEAL Study. Genet Med. 2004;6:192-196. 
26. Ormond KE, Haun J, Cook L, Duquette D, Ludowese C, Matthews AL. Recommendations for telephone counseling. J Genet Couns. 2000;9: 63-71.

27. Beck AT, Epstein N, Brown G, Steer RA. An inventory for measuring clinical anxiety: psychometric properties. J Consult Clin Psychol. 1988;56: 893-897.

28. Radloff LS. The CES-D scale: a self-report depression scale for research in the general population. App/ Psychol Meas. 1977;1:385-401.

29. Santor DA, Zuroff DC, Ramsay JO, Cervantes P, Palacios J. Examining scale discriminability in the BDI and CES-D as a function of depressive severity. Psychol Assess. 1995;7:131-139.

30. Horowitz M, Wilner N, Alvarez W. Impact of Event Scale: a measure of subjective stress. Psychosom Med. 1979;41:209-218.

31. Chung WW, Chen CA, Cupples LA, et al. A new scale measuring psychologic impact of genetic susceptibility testing for Alzheimer disease. Alzheimer Dis Assoc Disord. 2009;23:50-56.

32. Lipkus IM, Samsa G, Rimer BK. General performance on a numeracy scale among highly educated samples. Med Decis Making. 2001;21:37-44.

33. Fagerlin A, Zikmund-Fisher BJ, Ubel PA, Jankovic A, Derry H, Smith DM. Measuring numeracy without a math test: development of the Subjective Numeracy Scale (SNS). Med Decis Making. 2007;27:672-680.

34. Piaggio G, Elbourne DR, Altman DG, Pocock SJ, Evans SW. Reporting of noninferiority and equivalence randomized trials: an extension of the consort statement. JAMA. 2006;295:1152-1160.
35. Cooley K, Szczurko O, Perri D, et al. Naturopathic care for anxiety: a randomized controlled trial ISRCTN78958974. PLoS One. 2009;4:e6628.

36. Pearson-Fuhrhop KM, Dunn EC, Mortero S, et al. Dopamine genetic risk score predicts depressive symptoms in healthy adults and adults with depression. PLoS One. 2014;9:e93772.

37. McEvoy PM, Nathan P. Effectiveness of cognitive behavior therapy for diagnostically heterogeneous groups: a benchmarking study. J Consult Clin Psychol. 2007;75:344-350.

38. Locatis C, Williamson D, Gould-Kabler C, et al. Comparing in-person, video, and telephonic medical interpretation. J Gen Intern Med. 2010;25: 345-350.

39. Christensen KD, Roberts JS, Zikmund-Fisher BJ, et al. Associations between self-referral and health behavior responses to genetic risk information. Genome Med. 2015;7:10.

40. Vernarelli JA, Roberts JS, Hiraki S, Chen CA, Cupples LA, Green RC. Effect of Alzheimer disease genetic risk disclosure on dietary supplement use. Am J Clin Nutr. 2010;91:1402-1407.

41. Roberts JS, Christensen KD, Green RC. Using Alzheimer's disease as a model for genetic risk disclosure: implications for personal genomics. Clin Genet. 2011;80:407-414.

42. Robeznieks A. 'The right direction'. Mod Healthc. 2013;43:6-7.

43. Chatterjee N, Shi J, Garcia-Closas M. Developing and evaluating polygenic risk prediction models for stratified disease prevention. Nat Rev Genet. 2016;17:392-406. 